\title{
Uniportal video-assisted thoracic surgery for the treatment of lung cancer: a consensus report from Chinese Society for Thoracic and Cardiovascular Surgery (CSTCVS) and Chinese Association of Thoracic Surgeons (CATS)
}

\author{
Dingpei Han ${ }^{1}$, Yuqin Cao ${ }^{1}$, Han Wu ${ }^{1}$, Haifeng Wang ${ }^{2}$, Lei Jiang ${ }^{2}$, Deping Zhao ${ }^{2}$, Feng Yao ${ }^{3}$, Shuben $\mathrm{Li}^{4}$, \\ Chong Zhang ${ }^{5}$, Bin Zheng ${ }^{6}$, Junqiang Fan $^{7}$, Yongde Liao ${ }^{8}$, Bin Qiu', Fengwei Tan', Chun Chen ${ }^{6}$, \\ Yuming $\mathrm{Zhu}^{2}$, Shugeng $\mathrm{Gao}^{9}$, Hecheng $\mathrm{Li}^{1}$
}

${ }^{1}$ Department of Thoracic Surgery, Ruijin Hospital, Shanghai Jiao Tong University School of Medicine, Shanghai, China; ${ }^{2}$ Department of Thoracic Surgery, Shanghai Pulmonary Hospital, Tongji University School of Medicine, Shanghai, China; ${ }^{3}$ Department of Thoracic Surgery, Shanghai Chest Hospital, Shanghai Jiao Tong University, Shanghai, China; ${ }^{4}$ Department of Thoracic Surgery, The First Affiliated Hospital of Guangzhou Medical University, Guangzhou, China; ${ }^{5}$ Department of Thoracic Surgery, The First Affiliated Hospital, School of Medicine, Zhejiang University, Hangzhou, China; ${ }^{6}$ Department of Thoracic Surgery, Fujian Medical University Union Hospital, Fuzhou, China; ${ }^{7}$ Department of Thoracic Surgery, The Second Affiliated Hospital, School of Medicine, Zhejiang University, Hangzhou, China; ${ }^{8}$ Department of Thoracic Surgery, Union Hospital, Tongji Medical College, Huazhong University of Science and Technology, Wuhan, China; ${ }^{9}$ Department of Thoracic Surgery, Cancer Hospital, Chinese Academy of Medical Sciences and Peking Union Medical College, Beijing, China

Contributions: (I) Conception and design: D Han, C Chen, Y Zhu, S Gao, H Li; (II) Administrative support: H Wang, L Jiang, D Zhao, F Yao, S Li, C Zhang, B Zheng, J Fan, Y Liao, B Qiu, F Tan, C Chen, Y Zhu, S Gao, H Li; (III) Provision of study materials or patients: H Wang, L Jiang, D Zhao, F Yao, S Li, C Zhang, B Zheng, J Fan, Y Liao, B Qiu, F Tan, C Chen, Y Zhu, S Gao, H Li; (IV) Collection and assembly of data: D Han, Y Cao, H Wu; (V) Data analysis and interpretation: D Han, Y Cao, H Wu; (VI) Manuscript writing: All authors; (VII) Final approval of manuscript: All authors.

Correspondence to: Shugeng Gao, PhD. Department of Thoracic Surgery, Cancer Hospital, Chinese Academy of Medical Sciences and Peking Union Medical College, Beijing, China. Email: gaoshugeng@vip.sina.com; Hecheng Li, PhD. Department of Thoracic Surgery, Ruijin Hospital, Shanghai Jiao Tong University School of Medicine, Shanghai, China. Email: lihecheng2000@hotmail.com; Chun Chen, PhD. Department of Thoracic Surgery, Fujian Medical University Union Hospital, Fuzhou, China. Email: chenchun0209@163.com; Yuming Zhu, PhD. Department of Thoracic Surgery, Shanghai Pulmonary Hospital, Tongji University School of Medicine, Shanghai, China. Email: ymzhu2005@aliyun.com.

\begin{abstract}
Uniportal video-assisted thoracoscopic surgery (UniVATS) has been widely adopted in China, where several ultra-high volume thoracic surgical and training centers are located. The objective of this consensus from Chinese experts was to summarize the current application and give reference for the future development of UniVATS in the treatment of lung cancer. A panel of 41 experts from 21 Chinese hospitals was invited to join this project. The Delphi method was used in this consensus consisting of two rounds of voting. The questionnaire was based on the current clinical evidence. Forty (97.6\%) experts completed the 2 rounds of questionnaires. The experts' experience was relatively similar. We defined the UniVATS as monitor-dependent surgery, no use of rib-spreading and single incision less than $4 \mathrm{~cm}$. Tumor with stage of T1-T3 and N0-N2 is considered amenable to UniVATS. Other consensus was reached on several points outlining the safety and feasibility, surgical skills, learning curve, short-term and long-term outcomes for lung cancer, and current application of subxiphoid and nonintubated UniVATS approach. This consensus statement represents a collective agreement among Chinese experts to suggest that UniVATS is an effective alternative to multi-portal approach, although high-level evidence is expected in the future. Some agreements can be referred in the training of young surgeons.
\end{abstract}

Keywords: Consensus; lung cancer; uniportal video-assisted thoracoscopic surgery (UniVATS) 
Submitted May 09, 2020. Accepted for publication Jun 30, 2020.

doi: $10.21037 /$ tlcr-20-576

View this article at: http://dx.doi.org/10.21037/tlcr-20-576

\section{Introduction}

It has been more than a decade since uniportal videoassisted thoracoscopic surgery (UniVATS) was applied in thoracic surgery (1). Compared with the multi-portal videoassisted thoracoscopic surgery (VATS), UniVATS was related to less invasion and a vertical vision similar to open surgery (2). The first UniVATS lung cancer resection was described in 2010 (3). With the development of surgical skills and instruments, many complex thoracic procedures were reported to be performed by UniVATS approach (4-6).

Nowadays, UniVATS has been widely accepted and adopted in China but is less prevalent in Western countries, lack of high-quality evidence might be one of the reasons (7). In 2019, the Uniportal VATS Interest Group (UVIG) of the European Society of Thoracic Surgeons (ESTS) has published a consensus report on UniVATS for lobectomy (8), aiming to define and standardize the procedures, to optimize the indications and perioperative management, and to provide advice for future training. However, the largest quantity of UniVATS is performed in China, where several ultra-high volume thoracic surgical centers are located (9). Therefore, a consensus from Chinese experts will have a significance of reference in summarizing the current application of UniVATS in lung cancer. In this consensus, not only lobectomy, but also segmentectomy, subxiphoid and nonintubated approach were included. The recommendations from the experts will be helpful for the future development of UniVATS.

\section{Methods}

The Delphi method was implemented in the development of this consensus, which was initiated by Chinese Society for Thoracic and Cardiovascular Surgery (CSTCVS) and Chinese Association of Thoracic Surgeons (CATS). The characteristic and value of Delphi method in the field of health have been previously described (8). A total of 41 members from 21 Chinese hospitals were invited to participate in the 2 rounds of voting through questionnaire. All the experts possessed rich experience in both open and minimally invasive thoracic surgery, representing the topvolume surgical training and academic centers in China, most of them were the department directors or enjoyed the high reputation in field of thoracic surgery in China. The panel of the experts was identified by the corresponding authors of this manuscript.

The questionnaire was based on the current clinical evidence, including single choice, multiple choice, and survey questions. Each of the 2 rounds of voting was made through an online platform of questionnaire survey, evaluation and voting. The applet of the online platform was individually sent to the experts by WeChat (a social software) and was set to be opened only by the experts themselves. The first round of questionnaire was sent on $19^{\text {th }}$ June 2019 and was valid for a week. Anonymous results of the first round was attached to the second round of questionnaire sent on $28^{\text {th }}$ June 2019 , which was also valid for a week. A seminar on the consensus was held on $19^{\text {th }}$ August 2019 to reveal the anonymous results of the second round.

The results of the second round formed the basis of this consensus. The statistical analysis was finished by the online platform. Consensus was defined a priori as more than $50 \%$ agreement among the experts. The clinical practice was considered as "recommended (grade II)" if $50-74 \%$ of the experts reached an agreement and "highly recommended (grade I)" if $75 \%$ or more of the panel reached an agreement.

\section{Results}

A total of $40(97.6 \%)$ experts completed the 2 rounds of questionnaires. A summary of their institutions' case volume and time period of conducting UniVATS for lung cancer is shown in Figure 1. The experts' experience was relatively similar.

The definition of UniVATS is shown in Table 1. Fiftytwo point five experts make a 4-cm incision (grade II); $95 \%$ experts use an incision retractor (grade I); all the experts stand on the ventral side of the patient with their assistants on the opposite side $(62.5 \%$, grade II); $10-\mathrm{mm}$ camera is commonly used $(97.5 \%$, grade I) and fixed on the dorsal side of the incision (90\%, grade I); after surgery, only one chest tube (95\%, grade I) less than or equal $24-\mathrm{Fr}(75 \%$, grade I) is recommended to be placed in the same intercostal space 

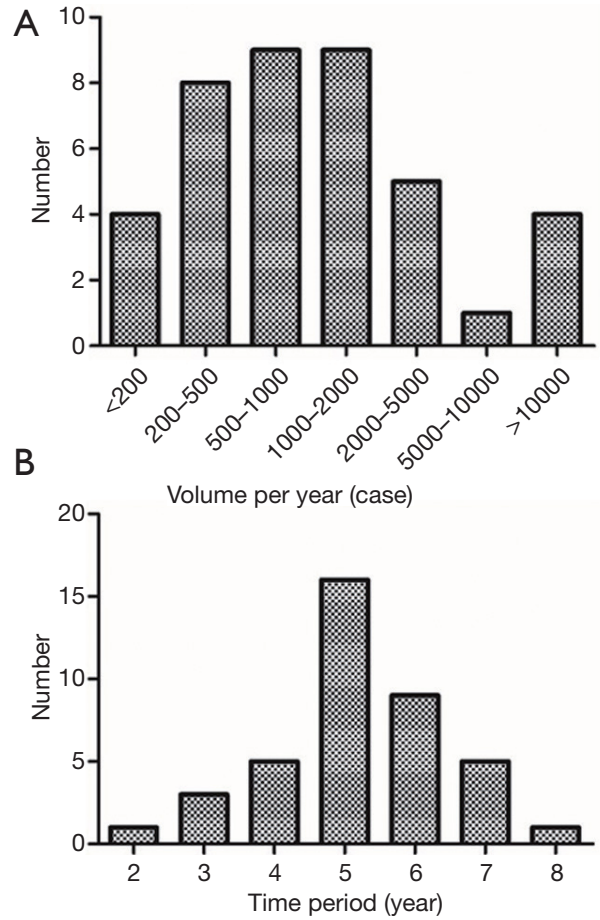

Figure 1 Surgical experience among the expert's institutions. (A) Distribution of surgical volume of UniVATS for lung cancer per year among the participating experts' institutions; (B) distribution of time period of UniVATS for lung cancer among the participating experts' institutions. UniVATS, uniportal videoassisted thoracoscopic surgery.

Table 1 Definition of UniVATS for lung resection

\begin{tabular}{lc}
\hline Question & $\mathrm{N}(\%)$ \\
\hline What would be the size of incision? & 0 \\
$2 \mathrm{~cm}$ & $18(45.0)$ \\
$3 \mathrm{~cm}$ & $21(52.5)$ \\
$4 \mathrm{~cm}$ & $1(2.5)$ \\
$5 \mathrm{~cm}$ & 0 \\
$>5 \mathrm{~cm}$ & \\
Do you use incision retractor? & $38(95.0)$ \\
Yes & $2(5.0)$ \\
No & \\
Which side do you fix the camera on? & $36(90.0)$ \\
Dorsal side of the incision & $2(5.0)$ \\
Ventral side of the incision & $2(5.0)$ \\
No fix
\end{tabular}

Table 1 (continued)
Table 1 (continued)

\begin{tabular}{lc}
\hline Question & $\mathrm{N}(\%)$ \\
\hline What type of camera do you use? & $1(2.5)$ \\
$5 \mathrm{~mm}$ & $39(97.5)$ \\
$10 \mathrm{~mm}$ & \\
Which side do you stand? & 0 \\
Dorsal side of the patient & $40(100.0)$ \\
Ventral side of the patient & \\
Which side do your assistant stand? & $13(32.5)$ \\
Same side & $25(62.5)$ \\
Opposite side & $2(5.0)$ \\
As appropriate
\end{tabular}

How many chest tubes placed after surgery?

1

2

What the size of chest tube placed after surgery?

$\begin{array}{lc}16 \mathrm{Fr} & 2(5.0) \\ 20 \mathrm{Fr} & 15(37.5) \\ 24 \mathrm{Fr} & 13(32.5) \\ 28 \mathrm{Fr} & 10(25.0)\end{array}$

How do you place the chest tube?

The same intercostal space through the incision

$39(97.3)$

Another intercostal space through the incision

Make another incision

0

Which side do you fix the chest tube?

Dorsal side of the incision

$30(75.0)$

Ventral side of the incision

Middle of the incision

$6(15.0)$

Do you use another inserted catheter (pig-tail catheter)?

Yes

20 (50.0)

No

$20(50.0)$

UniVATS, uniportal video-assisted thoracoscopic surgery.

through the incision (97.3\%, grade I) and fixed on the dorsal side of the incision ( $75 \%$, grade I); no other inserted catheter such as pig-tail catheter is recommended.

As demonstrated in Table 2, incision at $5^{\text {th }}$ intercostal space is recommended for UniVATS lobectomy of right 
Table 2 Site (intercostal space) of the incision for UniVATS

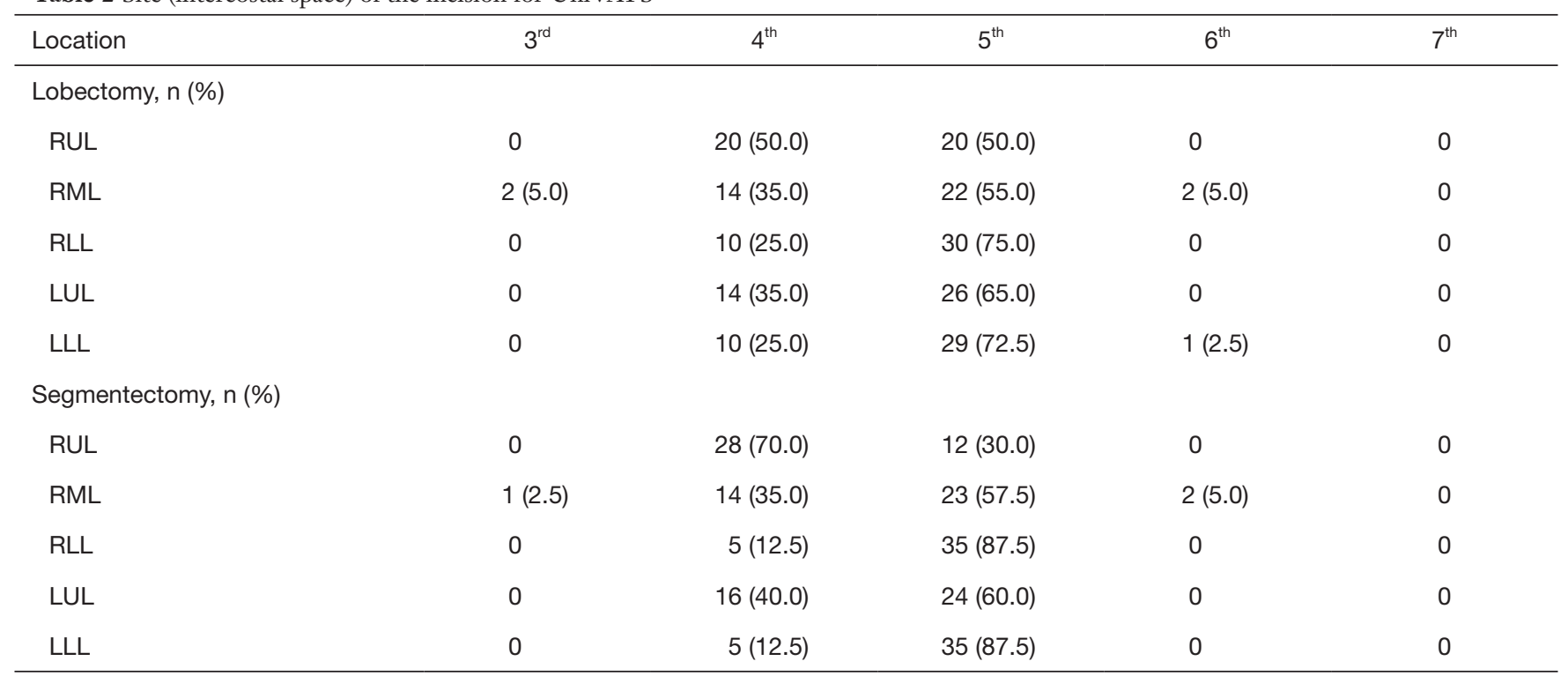

RUL, right upper lobe; RML, right middle lobe; RLL, right lower lobe; LUL, left upper lobe; LLL, left lower lobe; UniVATS, uniportal videoassisted thoracoscopic surgery.

Table 3 Placement of incision for UniVATS

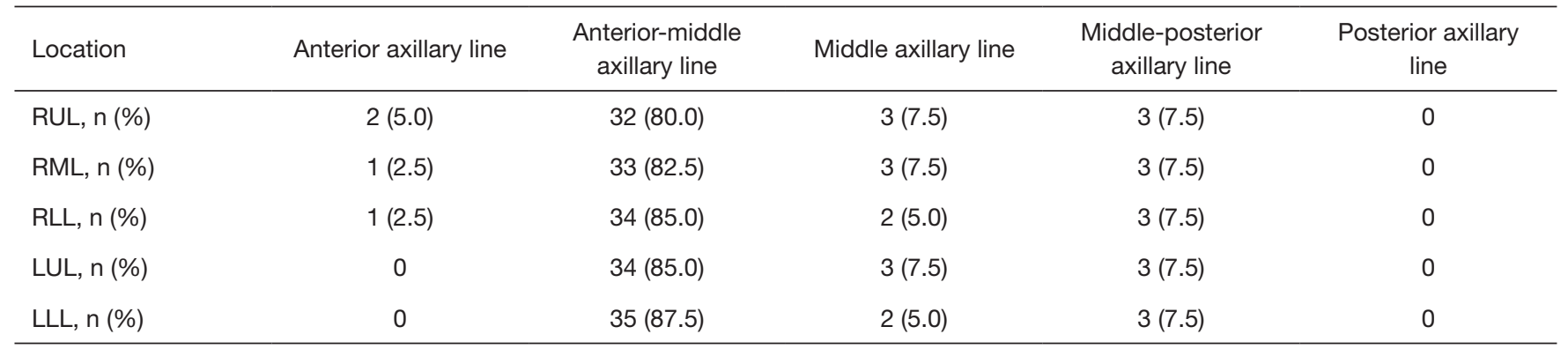

RUL, right upper lobe; RML, right middle lobe; RLL, right lower lobe; LUL, left upper lobe; LLL, left lower lobe; UniVATS, uniportal videoassisted thoracoscopic surgery.

middle lobe (RML) (55\%, grade II), right lower lobe (RLL) (75\%, grade I), left upper lobe (LUL) (65\%, grade II), and left lower lobe (LLL) (72.5\%, grade II); for UniVATS segmentectomy, incision at 5 th intercostal space is recommended in RML (57.5\%, grade II), RLL (87.5\%, grade I), LUL (60\%, grade II), and LLL (87.5\%, grade I), while incision at 4th intercostal space is recommended in right upper lobe (RUL) (70\%, grade II). As shown in Table 3, there is a strong recommendation of UniVATS at anteriormiddle axillary line ( $\geq 80 \%$ for all lobes, grade I).

A summary of experts' opinions regarding the eligibility of UniVATS for lung cancer is presented in Table 4. Tumor with stage of T1-T3 (60\%, grade II) and N0-N2 (77.5\%, grade I) is considered amenable to UniVATS; preoperative chemotherapy is not a contraindication $(85 \%$, grade I), while previous thoracic surgery or pleurisy $(57.5 \%$, grade II), tumor invading the hilar ( $90 \%$, grade I), and invasion of chest wall, phrenic nerve or pericardium ( $85 \%$, grade I) are considered as relative contraindications; regarding the indication of sleeve resection, only bronchus sleeve resection is recommended to be performed by UniVATS (62.5\%, grade II); according to this consensus, UniVATS is suitable for pneumonectomy ( $92.5 \%$, grade I) and is deemed feasible and safe for segmentectomy ( $97.5 \%$, grade I).

A summary of safety and feasibility of UniVATS for lung cancer is shown in Table 5. Calcified lymph nodes 
Table 4 Eligibility of UniVATS for lung cancer

\begin{tabular}{|c|c|}
\hline Question & $\mathrm{N}(\%)$ \\
\hline \multicolumn{2}{|l|}{ T stage } \\
\hline $\mathrm{T} 1$ & 0 \\
\hline $\mathrm{T} 1, \mathrm{~T} 2$ & $16(40.0)$ \\
\hline $\mathrm{T} 1, \mathrm{~T} 2, \mathrm{~T} 3(\leq 7 \mathrm{~cm})$ & $24(60.0)$ \\
\hline \multicolumn{2}{|l|}{$\mathrm{N}$ stage } \\
\hline NO & 0 \\
\hline No, N1 & $9(22.5)$ \\
\hline N0, N1, N2 & $31(77.5)$ \\
\hline \multicolumn{2}{|l|}{ Preoperative chemotherapy is } \\
\hline Absolute contraindication & 0 \\
\hline Relative contraindication & $6(15.0)$ \\
\hline Not a contraindication & $34(85.0)$ \\
\hline \multicolumn{2}{|l|}{ Preoperative radiotherapy is } \\
\hline Absolute contraindication & $10(25.0)$ \\
\hline Relative contraindication & $15(37.5)$ \\
\hline Not a contraindication & $15(37.5)$ \\
\hline \multicolumn{2}{|l|}{ Previous thoracic surgery/pleurisy is } \\
\hline Absolute contraindication & 0 \\
\hline Relative contraindication & $23(57.5)$ \\
\hline Not a contraindication & $17(42.5)$ \\
\hline \multicolumn{2}{|l|}{ Tumor invading the hilar structure is } \\
\hline Absolute contraindication & $1(2.5)$ \\
\hline Relative contraindication & $36(90.0)$ \\
\hline Not a contraindication & $3(7.5)$ \\
\hline \multicolumn{2}{|l|}{$\begin{array}{l}\text { Tumor invading chest wall, phrenic nerve or } \\
\text { pericardium is }\end{array}$} \\
\hline Absolute contraindication & $3(7.5)$ \\
\hline Relative contraindication & $34(85.0)$ \\
\hline Not a contraindication & $3(7.5)$ \\
\hline \multicolumn{2}{|l|}{ Indication of sleeve resection } \\
\hline Bronchus sleeve and sleeve angioplasty & $13(32.5)$ \\
\hline Bronchus sleeve & $25(62.5)$ \\
\hline Sleeve angioplasty & 0 \\
\hline Not for all & $2(5.0)$ \\
\hline
\end{tabular}

Table 4 (continued)
Table 4 (continued)

\begin{tabular}{lc}
\hline Question & $\mathrm{N}(\%)$ \\
\hline Whether UniVATS is suitable for & \\
pneumonectomy? & $37(92.5)$ \\
Yes & $3(7.5)$ \\
No & \\
UniVATS segmentectomy is safety and \\
feasible \\
Agree \\
Neither agree nor disagree \\
Disagree
\end{tabular}

UniVATS, uniportal video-assisted thoracoscopic surgery.

(70\%, grade II), extensive pleural adhesions (65\%, grade II), technical difficulties (70\%, grade II), and massive hemorrhage $(95 \%$, grade I) are the main reasons for auxiliary operating ports, while calcified lymph nodes $(60 \%$, grade II) and massive hemorrhage ( $92.5 \%$, grade I) are the dominant reasons for conversion; most experienced experts would try to deal with pulmonary vascular branch bleeding (97.5\%, grade I) or tracheobronchial injury $(92.5 \%$, grade I) by UniVATS, and they agree on the absence of difference between UniVATS and multi-portal VATS in terms of mortality and intraoperative adverse events according to the current literature (92.5\%, grade I).

As shown in Table 6, most experts need 2 assistants ( $60 \%$, grade II) with higher capability $(97.5 \%$, grade I); both the site of incision (100\%, grade I) and the capability of assistant (100\%, grade I) can influence the processing fluency of UniVATS; systematic lymph nodes dissection is recommended for lobectomy $(92.5 \%$, grade I) while systematic lymph nodes sampling is recommended for segmentectomy (75\%, grade I) in UniVATS after lung resection $(85 \%$, grade I); concerning the current evidence, the experts agree that there is no difference between UniVATS and multi-portal approach in the quantity of lymph nodes dissection $(92.5 \%$, grade I); paravertebral intercostal nerve block $(62.5 \%$, grade II) is not recommended after UniVATS procedure.

Regarding the learning curve of UniVATS (Table 7), 30 cases are deemed the cut-off point both for lobectomy $(70 \%$, grade II) and segmentectomy $(72.5 \%$, grade II); operative time $(87.5 \%$, grade I) and perioperative complications $(87.5 \%$, grade I) are two recommended evaluation indices of learning curve for UniVATS. Surgeons should perform 
Table 5 The safety and feasibility of UniVATS for lung cancer

\begin{tabular}{|c|c|}
\hline Question & N (\%) \\
\hline \multicolumn{2}{|c|}{$\begin{array}{l}\text { Under which situations would you add another } \\
\text { port? }\end{array}$} \\
\hline Extensive pleural adhesions & $26(65.0)$ \\
\hline Calcified lymph nodes & $28(70.0)$ \\
\hline Absence of fissure & $3(7.5)$ \\
\hline Poor lung deflation & $12(30.0)$ \\
\hline Involvement of nerves or pericardium & $15(37.5)$ \\
\hline Poor assistant cooperation & $20(50.0)$ \\
\hline Other technical difficulties & $28(70.0)$ \\
\hline Massive hemorrhage & $38(95.0)$ \\
\hline None of above & 0 \\
\hline \multicolumn{2}{|c|}{$\begin{array}{l}\text { Under which situations would you convert to } \\
\text { open? }\end{array}$} \\
\hline Extensive pleural adhesions & $10(25.0)$ \\
\hline Calcified lymph nodes & $24(60.0)$ \\
\hline Absence of fissure & $1(2.5)$ \\
\hline Poor lung deflation & $5(12.5)$ \\
\hline Involvement of nerves or pericardium & $13(32.5)$ \\
\hline Poor assistant cooperation & $8(20.0)$ \\
\hline Other technical difficulties & $21(52.5)$ \\
\hline Massive hemorrhage & $37(92.5)$ \\
\hline None of above & 0 \\
\hline \multicolumn{2}{|c|}{$\begin{array}{l}\text { Under which situations would you try to deal } \\
\text { with by UniVATS? }\end{array}$} \\
\hline Pulmonary vascular trunk bleeding & $22(55.0)$ \\
\hline Pulmonary vascular branch bleeding & $39(97.5)$ \\
\hline Tracheobronchial injury & $37(92.5)$ \\
\hline \multicolumn{2}{|c|}{$\begin{array}{l}\text { Only surgeons with rich experience can try to } \\
\text { deal with massive hemorrhage by UniVATS }\end{array}$} \\
\hline Agree & $38(95.0)$ \\
\hline Neither agree nor disagree & $2(5.0)$ \\
\hline Disagree & 0 \\
\hline \multicolumn{2}{|c|}{$\begin{array}{l}\text { No differences between UniVATS and multi- } \\
\text { portal VATS in terms of mortality, incidence of } \\
\text { intraoperative adverse events }\end{array}$} \\
\hline Agree & $37(92.5)$ \\
\hline Neither agree nor disagree & $3(7.5)$ \\
\hline Disagree & 0 \\
\hline
\end{tabular}

UniVATS, uniportal video-assisted thoracoscopic surgery.
Table 6 Surgical skills of UniVATS for lung cancer

\begin{tabular}{|c|c|}
\hline Question & $\mathrm{N}(\%)$ \\
\hline \multicolumn{2}{|l|}{ How many assistants do you need? } \\
\hline 1 & $16(40.0)$ \\
\hline 2 & $24(60.0)$ \\
\hline 3 & 0 \\
\hline \multicolumn{2}{|l|}{$\begin{array}{l}\text { How is the capability requirement for } \\
\text { assistant? }\end{array}$} \\
\hline UniVATS requires higher capability & $39(97.5)$ \\
\hline Multi-portal VATS requires higher capability & $1(2.5)$ \\
\hline No difference & 0 \\
\hline \multicolumn{2}{|l|}{$\begin{array}{l}\text { The site of incision is an influence on the } \\
\text { procedure }\end{array}$} \\
\hline Yes & $40(100.0)$ \\
\hline No & 0 \\
\hline \multicolumn{2}{|l|}{$\begin{array}{l}\text { The capability of assistant is an influence on } \\
\text { the procedure }\end{array}$} \\
\hline Yes & $40(100.0)$ \\
\hline No & 0 \\
\hline \multicolumn{2}{|l|}{$\begin{array}{l}\text { Which is the proper management of lymph } \\
\text { nodes in lobectomy? }\end{array}$} \\
\hline Systematic lymph nodes dissection & $37(92.5)$ \\
\hline Lobe-specific lymph nodes dissection & $2(5.0)$ \\
\hline Systematic lymph nodes sampling & $1(2.5)$ \\
\hline Lobe-specific sampling & 0 \\
\hline Random/no sampling & 0 \\
\hline \multicolumn{2}{|l|}{$\begin{array}{l}\text { Which is the proper management of lymph } \\
\text { nodes in segmentectomy? }\end{array}$} \\
\hline Systematic lymph nodes dissection & $1(2.5)$ \\
\hline Lobe-specific lymph nodes dissection & $5(12.5)$ \\
\hline Systematic lymph nodes sampling & $30(75.0)$ \\
\hline Lobe-specific sampling & $4(10.0)$ \\
\hline Random/no sampling & 0 \\
\hline \multicolumn{2}{|l|}{$\begin{array}{l}\text { Which is the proper management of lymph } \\
\text { nodes? }\end{array}$} \\
\hline $\begin{array}{l}\text { Lung resection before lymph nodes } \\
\text { dissection }\end{array}$ & $34(85.0)$ \\
\hline $\begin{array}{l}\text { Lymph nodes dissection before lung } \\
\text { resection }\end{array}$ & $6(15.0)$ \\
\hline
\end{tabular}

Table 6 (continued) 
Table 6 (continued)

\begin{tabular}{lc}
\hline Question & $\mathrm{N}(\%)$ \\
\hline The quantity of lymph nodes harvested is? & 0 \\
More by UniVATS & $2(5.0)$ \\
More by multi-portal approach & $37(92.5)$ \\
No difference between the two approaches & $1(2.5)$ \\
Not sure & \\
Do you perform paravertebral intercostal nerve & \\
block after surgery? & $15(37.5)$ \\
Yes & $25(62.5)$ \\
No
\end{tabular}

UniVATS, uniportal video-assisted thoracoscopic surgery.

at least 50 cases annually to maintain uniportal operative skills $(60 \%$, grade II), and some training programs such as video training $(82.5 \%$, grade I) and long-term advanced study $(82.5 \%$, grade I) are highly recommended for young surgeons; in addition, the experts' scores evaluating difficulty in lobectomy are ranked as follows (from complex to simple): LUL, RML, RLL, RUL, and LLL.

Regarding the short-term and long-term outcomes of UniVATS for lung cancer (Table 8), the experts reach an agreement on the absence of significant difference in postoperative drainage duration and length of stay $(87.5 \%$, grade I), overall complications and pulmonary complications (90\%, grade I), and overall survival and disease free survival (90\%, grade I) between UniVATS and multi-portal approach according to the current evidence; UniVATS shows a lower postoperative pain score $(52.5 \%$, grade II), a better emotional and functional status and a higher quality of life $(62.5 \%$, grade II) than multi-portal approach.

Base on the current literature, as shown in Table 8, the experts agree that subxiphoid UniVATS can reduce intercostal nerve injury and postoperative pain $(90.9 \%$, grade I), but is not recommended for complex procedure (81.8\%, grade I); nonintubated UniVATS can be applied only on the condition of experienced medical team and rigorous selection of patients ( $80 \%$, grade I).

Other survey results are listed in Table 9. The experts suggest conducting randomized controlled trials about UniVATS to obtain high-level evidence (95\%, grade I); the experts agree that UniVATS has benefited from the improvement of surgical instruments $(92.5 \%$, grade I) and serves as a promising direction of minimally invasive
Table 7 The learning curve of UniVATS for lung cancer

\begin{tabular}{|c|c|}
\hline Question & $\mathrm{N}(\%)$ \\
\hline \multicolumn{2}{|l|}{$\begin{array}{l}\text { How many cases are required for the learning } \\
\text { curve of lobectomy? }\end{array}$} \\
\hline About 30 & $28(70.0)$ \\
\hline About 50 & $12(30.0)$ \\
\hline About 70 & 0 \\
\hline About 100 & 0 \\
\hline \multicolumn{2}{|l|}{$\begin{array}{l}\text { How many cases are required for the learning } \\
\text { curve of segmentectomy? }\end{array}$} \\
\hline About 30 & $29(72.5)$ \\
\hline About 50 & $10(25.0)$ \\
\hline About 70 & 0 \\
\hline About 100 & $1(2.5)$ \\
\hline \multicolumn{2}{|l|}{ What do you think about the learning curve? } \\
\hline Uniportal approach is shorter & $2(5.0)$ \\
\hline Multi-portal approach is shorter & $13(32.5)$ \\
\hline No difference between the two approaches & $25(62.5)$ \\
\hline Not sure & 0 \\
\hline \multicolumn{2}{|l|}{$\begin{array}{l}\text { Operative time is an evaluation index of } \\
\text { learning curve }\end{array}$} \\
\hline Agree & $35(87.5)$ \\
\hline Neither agree nor disagree & $5(12.5)$ \\
\hline Disagree & 0 \\
\hline \multicolumn{2}{|l|}{$\begin{array}{l}\text { Perioperative complication is an evaluation } \\
\text { index of learning curve }\end{array}$} \\
\hline Agree & $35(87.5)$ \\
\hline Neither agree nor disagree & $5(12.5)$ \\
\hline Disagree & 0 \\
\hline \multicolumn{2}{|l|}{$\begin{array}{l}\text { The experience of thoracotomy or multi-portal } \\
\text { approach would affect the learning curve in } \\
\text { young surgeons }\end{array}$} \\
\hline Agree & $19(47.5)$ \\
\hline Neither agree nor disagree & $15(37.5)$ \\
\hline Disagree & $6(15.0)$ \\
\hline \multicolumn{2}{|l|}{$\begin{array}{l}\text { The rank of lobectomy according to the } \\
\text { difficulty (score) }\end{array}$} \\
\hline Left upper lobe & 4.48 \\
\hline Right middle lobe & 3.55 \\
\hline
\end{tabular}

Table 7 (continued) 
Table 7 (continued)

\begin{tabular}{lc}
\hline Question & $\mathrm{N}(\%)$ \\
\hline Right lower lobe & 2.35 \\
Right upper lobe & 2.30 \\
Left lower lobe & 2.28 \\
How many cases are required for a surgeon to & \\
maintain the skills? & \\
About 25 & $5(12.5)$ \\
About 50 & $24(60.0)$ \\
About 75 & 0 \\
About 100 & $11(27.5)$ \\
Which way of training do you recommend to & \\
young surgeon? & \\
Self-determination training & $24(60.0)$ \\
Video training & $33(82.5)$ \\
Consultation of experts & $15(37.5)$ \\
Animal experiment & $21(52.5)$ \\
Simulator & $25(62.5)$ \\
Webcast learning & $27(67.5)$ \\
\hline UnivATS, uniportal video-assisted thoracoscopic surgery. & $33(82.5)$ \\
\hline
\end{tabular}

thoracic surgery (80\%, grade I) and an effective part of enhance recovery after surgery for lung cancer $(77.5 \%$, grade II).

\section{Discussion}

\section{Definition of UniVATS for lung resection}

According to the consensus of the experts, we defined the UniVATS as "monitor-dependent surgery, no use of rib-spreading and single incision less than $4 \mathrm{~cm}$ ". Most experts agreed that the maximum size of incision should be less than $4 \mathrm{~cm}$. Considering the fact that $97.5 \%$ of the experts used $10-\mathrm{mm}$ camera, incision less than $3 \mathrm{~cm}$ might be gradually adopted owing to the popularization of slim camera and instruments $(10,11)$. An anterior-middle axillary incision at $5^{\text {th }}$ intercostal space was recommended by most of the experts for both lobectomy and segmentectomy, there were also reports on different locations of incision such as subaxillary, transcervical, and posterior axillary incision (12-14). Moreover, in order to prevent adverse events
Table 8 Outcomes of UniVATS for lung cancer \& other UniVATS approaches

\begin{tabular}{lc}
\hline Question & $\mathrm{N}(\%)$ \\
\hline $\begin{array}{l}\text { There is no significant difference in postoperative } \\
\text { drainage duration and length of stay between }\end{array}$ \\
UniVATS and multi-portal approach \\
Agree & $35(87.5)$ \\
Neither agree nor disagree & 0 \\
Disagree & $5(12.5)$ \\
Postoperative pain score in UniVATS is lower & 0 \\
than in multi-portal approach (lower is better) & \\
Agree & $21(52.5)$ \\
Neither agree nor disagree & $2(5.0)$ \\
Disagree & $17(42.5)$
\end{tabular}

UniVATS shows a better emotional and functional status and a higher quality of life than multi-portal approach

$$
\begin{aligned}
& \text { Agree } \\
& \text { Neither agree nor disagree } \\
& \text { Disagree }
\end{aligned}
$$

There is no significant difference in overall complications and pulmonary complications between UniVATS and multi-portal approach

Agree

$36(90.0)$

Neither agree nor disagree

0

Disagree

UniVATS and multi-portal approach show no difference in survival rate as the treatment of early stage lung cancer

$\begin{array}{lc}\text { Agree } & 36(90.0) \\ \text { Neither agree nor disagree } & 4(10.0) \\ \text { Disagree } & 0 \\ \text { Subxiphoid UniVATS can reduce intercostal } \\ \text { nerve injury and pain }{ }^{a} \\ \text { Agree } & 10(90.9) \\ \text { Neither agree nor disagree } & 1(9.1) \\ \text { Disagree } & 0 \\ \text { Subxiphoid UniVATS is not recommended for } \\ \text { complex procedure }\end{array}$

Table 8 (continued) 
Table 8 (continued)

\begin{tabular}{lc}
\hline Question & $\mathrm{N}(\%)$ \\
\hline $\begin{array}{l}\text { Nonintubated UniVATS can only be applied on } \\
\text { condition of experienced medical team and } \\
\text { rigorous selection of patients }\end{array}$ \\
Agree & $32(80.0)$ \\
Neither agree nor disagree & $6(15.0)$ \\
Disagree & $2(5.0)$ \\
\hline
\end{tabular}

a, eleven experts finished this question. UniVATS, uniportal video-assisted thoracoscopic surgery.

such as air or fluid leakage and impaired reconstruction of muscular layer, some surgeons proposed feasible methods for chest tube placement and incision closing $(15,16)$. these attempts accompanied by different advantage or disadvantages reflected the effort made by surgeons to improving the procedure of UniVATS.

\section{Eligibility of UniVATS for lung cancer}

According to the results, UniVATS lung resection was recommended for tumor stage $\mathrm{T} 1-\mathrm{T} 3$ and $\mathrm{N} 0-\mathrm{N} 2$. It was usually believed that tissue adhesions and increased vascular fragility after neoadjuvant chemotherapy could increase the risk of surgery, but some recent studies suggested that preoperative chemotherapy had no effects on operative time, intraoperative complications, and lymph nodes dissection $(17,18)$. So as showed in this consensus, preoperative chemotherapy was no longer a contraindication to UniVATS for lung resection. However, no consensus was reached on preoperative radiotherapy due to lack of relevant evidence.

With the development of endoscopic instruments and surgical skills, experienced surgeons could perform complex operations by multi-portal VATS including sleeve resection and pneumonectomy with similar results compared to open surgery (19-21). According to this consensus, UniVATS was also suitable for bronchus sleeve resection and pneumonectomy (22-28). However, as case report and surgical techniques dominated the types of publication, the feasibility and safety of UniVATS on sleeve resection and pneumonectomy should be verified by future studies.

UniVATS shared the same indications with multi-portal VATS for segmentectomy to treat lung cancer. Recent studies suggested that UniVATS segmentectomy was associated with less blood loss, shorter operative time and
Table 9 Other survey

\begin{tabular}{|c|c|}
\hline Question & $\mathrm{N}(\%)$ \\
\hline \multicolumn{2}{|c|}{$\begin{array}{l}\text { The progress of UniVATS had benefited from the } \\
\text { improvement of surgical instruments }\end{array}$} \\
\hline Agree & $37(92.5)$ \\
\hline Neither agree nor disagree & $2(5.0)$ \\
\hline Disagree & $1(2.5)$ \\
\hline \multicolumn{2}{|c|}{$\begin{array}{l}\text { UniVATS would be a development direction of } \\
\text { minimally invasive thoracic surgery }\end{array}$} \\
\hline Agree & $32(80.0)$ \\
\hline Neither agree nor disagree & $8(20.0)$ \\
\hline Disagree & 0 \\
\hline \multicolumn{2}{|c|}{$\begin{array}{l}\text { Is it necessary to conduct a randomized } \\
\text { controlled trial about UniVATS? }\end{array}$} \\
\hline Yes & $38(95.0)$ \\
\hline No & $2(5.0)$ \\
\hline \multicolumn{2}{|c|}{$\begin{array}{l}\text { UniVATS is an effective part of enhance recovery } \\
\text { after surgery for lung cancer }\end{array}$} \\
\hline Agree & $31(77.5)$ \\
\hline Neither agree nor disagree & $8(20.0)$ \\
\hline Disagree & $1(2.5)$ \\
\hline
\end{tabular}

UniVATS, uniportal video-assisted thoracoscopic surgery.

faster recovery compared with multi-portal VATS $(4,29,30)$. Based on the current evidence, the experts agreed that UniVATS was feasible and safe for segmentectomy. The key procedures of UniVATS segmentectomy included location of nodules, selection and placement of instruments, and identification of intersegmental planes. Novel techniques such as three-dimensional reconstruction, magnetic navigation, and fluorochrome staining would facilitate the operation in both UniVATS and multi-portal VATS approach $(31,32)$.

\section{Safety and feasibility of UniVATS for lung cancer}

According to the consensus, calcified lymph was a key point of UniVATS procedures, calcified nodes fused to the hilum, fissure and bronchus might lead to an accident during the procedure. It was reported that $41 \%$ of conversions in VATS lobectomy were due to hilar calcified nodes, which was the leading cause of intraoperative bleeding (33). Early studies suggested that the risk of intraoperative bleeding in VATS 
was higher than that in thoracotomy, especially in the initial stage of learning curve (34). However, a recent study with a large sample size revealed no difference in intraoperative complication rate between VATS and thoracotomy $(1.57 \%$ vs. $1.44 \%, \mathrm{P}=1.0$ ) (35). Just like during the procedures of multi-portal VATS, the successful management of intraoperative complications by UniVATS was also related to the severity of complication, surgeons' experience, surgical instruments, and the cooperation of assistants (36). An experienced team could try to deal with severe hemorrhage by UniVATS (37), while doctors in the learning stage needed to be familiar with anatomy, keep calm, and prepare for timely conversion to thoracotomy (38). So, agreement was reached by the experts that experienced surgeons could try to deal with intraoperative complications such as severe hemorrhage, vascular branch bleeding or tracheobronchial injury by UniVATS. Some experienced surgeons have shared their surgical skills to deal with calcified lymph nodes (39), which was no longer considered as a contraindication to both multi-portal VATS and UniVATS.

Some retrospective studies on UniVATS have reported the mortality, the rate of conversion, and the intraoperative complication rate as $3.3 \%(40), 4.6 \%$, and $5.6 \%(41)$, respectively. A retrospective literature review (40) implied no difference between uniportal and multi-portal VATS in operation time and intraoperative blood loss. Based on the current evidence, the experts agreed that there was no difference between uniportal and multi-portal approach in terms of intraoperative adverse events, rate of conversion to thoracotomy, and mortality.

\section{Surgical techniques of UniVATS for lung cancer}

UniVATS usually required one or two assistants, but it was more technically demanding for assistants than traditional thoracoscopy. In case of only one assistant available, the proficiency of the assistant would have a great impact on the procedure of UniVATS, a successful UniVATS is dependent on the tacit cooperation of the surgeon and the assistant, especially in emergency conditions (42). In addition, the location of incision would also greatly impact the procedure of UniVATS according the experts' opinions.

As mentioned in the results, systematic lymph node dissection was recommended for UniVATS lobectomy while systematic lymph node sampling was recommended for UniVATS segmentectomy. Regarding the number of lymph nodes dissection, evidence have showed controversial results between multi-portal VATS and open surgery $(43,44)$. The similar situation was presented between UniVATS and multi-portal VATS, some reports indicated no difference between uniportal and multi-portal VATS, but some others suggested that uniportal approach yielded more lymph nodes than multi-portal approach did (40). Although the experts in this consensus agreed that there was no difference between UniVATS and multi-portal approach in the quantity of lymph nodes dissection, the conclusion should be verified by future high-level evidence.

In order to optimize the surgical procedure, many surgeons have implemented some exploratory techniques of lymph node dissection in UniVATS as followed: Lateral prone position was used to improve the efficiency of mediastinal lymph node dissection (45); non-grasping technology was considered to reduce the interference of surgical instruments and to clarify the surgical field: the suction could not only eliminate the smoke produced by electric hook or ultrasonic knife and the bleeding or exudate caused by separating procedure, but also had the role of blunt dissection and traction without clamping lymph nodes, which could minimize the fracture of lymph nodes and consequent bleeding (46). In addition, reduced operation time and intraoperative blood loss were reported in a modular pattern of UniVATS lymphadenectomy, in which the lymph nodes were resected in the following order: (I) right lung: dissection of group 7, 8, 9, 10 lymph nodes, followed by lung resection and finally the dissection of upper mediastinal lymph nodes; (II) left lung: dissection of group 8, 9, 7, 10, 5, 4L, 6 lymph nodes, and then lung resection (47).

Although the surgical techniques mentioned above were mostly based on the clinical experience of each center and lacked high quality evidence, the experts' consensus might provide young surgeons with some references.

\section{Learning curve of UniVATS for lung cancer}

Learning curve was generated to evaluate the surgical proficiency of surgeons by comparing the changes of perioperative indices, which reflected not only the competence of surgeons, but also the complexity of the procedure. According to the current evidence, the cutoff value in the learning curve of UniVATS for lobectomy varied from 25 to 40 cases (48-51), and the consensus gave a recommendation of 30 cases. The cut-off point in the learning curve of UniVATS segmentectomy was reported as 33 cases by a sole study (52) and recommended as 30 
cases by the experts. Similarly, the cut-off point was 27 cases in the learning curve of multi-portal VATS for lung resection (53). So, this consensus suggested that there was no difference between uniportal and multi-portal VATS in terms of learning curve.

Operation time was considered as an important evaluation index of learning curve in this consensus, it was significantly reduced after finishing the learning curve in both lobectomy and segmentectomy according to the above studies. In addition, postoperative hospital stay, drainage duration, intraoperative bleeding, conversion rate, operative mortality, and complication rate were also reported as indicators in the learning curve for UniVATS (48). According to this consensus, the experts agreed that postoperative complication rate was another ideal evaluation index of learning curve for UniVATS.

The basic skills of young surgeons were usually considered as an influence on the learning curve. However, the experts did not reach an agreement that the experience of thoracotomy or multi-portal VATS would affect the learning curve of UniVATS. For young doctors, video training, long-term advanced study, self-determination training, training on simulator, and webcast learning were recommended by the experts. In addition, according to the experts' scores, the rank of difficulty in UniVATS lobectomy was as LUL, RML, RLL, RUL, and LLL (from complex to simple), serving as a reference for young trainees.

\section{Short-term and long-term outcomes of UniVATS for lung cancer}

According to a systematic review of published evidence (40), most studies showed no difference in length of stay and drainage duration after surgery between UniVATS lobectomy and multi-portal approach. According to a meta-analysis involving 4,635 participants from 39 studies, UniVATS held advantages over conventional VATS in postoperative pain, length of stay, and duration of chest drain (54). Although the current evidence showed disputed results, the experts agreed that there was no significant difference in postoperative drainage duration and length of stay between UniVATS and multi-portal VATS, but UniVATS reported lower pain scores after surgery.

In terms of quality of life, retrospective studies supported that the small incision of UniVATS caused less hyperalgesia, numbness and allodynia after lobectomy than the multiportal approach, thus leading to a higher satisfaction with incision (55). The European Organization for Research and Treatment of Cancer Quality of Life Questionnaire (EORTC QLQ-C30) and the supplemental questionnaire QLQ-LC13 were adopted in another study where UniVATS indicated advantages over multi-portal VATS in functional status (physical function, role function, emotional function, and social function) and overall health condition at 2, 4 and 8 weeks after surgery (56). The panel of experts agreed that patients undergoing UniVATS showed better emotional and functional status after surgery and had a higher quality of life than those undergoing multi-portal VATS.

Complication rates after UniVATS ranged from 3\% to $40 \%$ in different publications (40). Such a discrepancy was associated with the sample size and type of complications as well. Studies with a larger sample size $(>100)$ reported $4-14 \%$ postoperative complications (41,57-62), among which prolonged air leak, pulmonary infection, and atelectasis were the leading ones. A meta-analysis with the highest quality of evidence reported no significant difference in postoperative complication rates between UniVATS and multi-incisional VATS [odds ratio $(\mathrm{OR})=0.80$, 95\% confidence interval (CI): 0.59-1.08, $\mathrm{P}=0.15$ ] (54). Several published comparative studies included pulmonary complications reported that incidence rates were reported as $5.1-17.8 \%$ after UniVATS and $4-20 \%$ after multiportal VATS $(55,62-66)$, indicating no significant difference between two approaches. The experts reached an agreement on the absence of difference in the incidence of overall complications and pulmonary complications between UniVATS and multi-portal VATS.

Regarding long-term outcomes, a retrospective study reported a 2 -year survival rate of $96 \%$ for stage I lung cancer and $83 \%$ for stage II lung cancer after UniVATS major lung resections (60). According to another observational study, the 3 -year overall survival rate of stage IA and IB lung cancer was $93.2 \%, 93.7 \%$, and $87.3 \%$ in single-port, two-port, and three-port VATS group, respectively. Statistical difference was neither observed in overall survival nor recurrence-free survival (61). In a recent study, the 5 -year overall survival rate was $80.1 \%$ for stage I lung cancer treated by UniVATS lobectomy, the 4-year progression-free survival rate of UniVATS segmentectomy was $94.1 \%$ (67). Agreement was reached by the experts that UniVATS and multi-portal VATS showed no difference in survival rate as the treatment of early stage lung cancer.

\section{Subxiphoid UniVATS approach for lung cancer}

This innovative approach was first applied in the thymectomy 
in 2012 (68) and in 2014 was reported the first subxiphoid UniVATS lobectomy (69). So far, UniVATS via subxiphoid approach has been applied in segmentectomy (70), synchronous bilateral segmentectomy (71), hilar calcified lymph nodes resection (72), and other complex surgeries. Regarding the safety, in a case series of 172 subxiphoid lobectomies, the conversion rate was $4.1 \%$ to open surgery and $2.9 \%$ to multi-portal approach, mortality was $0 \%$ and morbidity was $10.5 \%$ (73). Compared with conventional intercostal incision, the advantage of subxiphoid approach lied in reducing damage to intercostal nerve and postoperative pain. It was reported in a retrospective study that the pain scores from the first day after surgery to discharge were significantly lower in the group of subxiphoid UniVATS than in the group of intercostal UniVATS (74). However, the limited number of studies were mostly published by a same center, thus appealing for further evidence for the safety and feasibility of UniVATS via subxiphoid approach. There was no strong agreement in the safety and feasibility of subxiphoid UniVATS. The experts agreed that the subxiphoid UniVATS approach could reduce intercostal nerve injury and postoperative pain, but it was not recommended for complex lung surgeries.

\section{Nonintubated UniVATS approach for lung cancer}

For the sake of less invasive procedure, thoracic surgeons and anesthetists have tried to perform nonintubated thoracic surgeries. The earliest awake thoracoscopic lung surgery was reported in 2004 (75), and nonintubation was first combined with UniVATS in 2010 (76). It was reported in a series of 40 nonintubated UniVATS cases that the conversion rate was $7.5 \%$ to intubation and $2.5 \%$ to multi-portal VATS. The postoperative complication rate was $17.5 \%$, which was higher than the reported rate of $3-6 \%$ in nonintubated multi-portal VATS (77). According to another cohort of 188 cases, the conversion rate was $1.6 \%$ to intubation and $2.7 \%$ to multi-portal approach, followed by a complication rate of $8.5 \%$ after surgery (78). In another study on lung biopsy in patients with interstitial lung diseases, the conversion to 2-portal rate was $7.0 \%$ due to extensive pleural adhesion (79). Current publications of safety and feasibility in nonintubated VATS, either uniportal or multi-portal, were mainly case reports and case series. The lack of prospective clinical trials and the high demand for anesthetic team had limited the development of nonintubated UniVATS (80). Multidisciplinary team should implement strict controls over contraindications, including inexperienced team, difficulty in airway management, obesity, persistent cough, previous surgery, coagulation disorder, and neurologic symptoms (81). Nonintubated UniVATS represented the thoracic surgeons' constant pursuit of minimal invasion and revealed potentials for reducing costs as well as replacing some conventional surgeries by outpatient operations. Agreement was reached by the experts that nonintubated UniVATS could be applied in anatomical pulmonary resection on the condition of experienced medical team and rigorous selection of patients.

\section{Conclusions}

Above all, the consensus reported the definition and eligibility of UniVATS. The panel of experts also gave consentaneous opinions on some disputed questions based on present evidence. Compared with the consensus published by ESTS (8), this consensus reported similar results on most issues except the indication of UniVATS, extending from T2/N1 in the ESTS consensus to T3/N2 in this Chinese consensus, although the long-term outcomes should be evaluated by further studies. Moreover, this consensus presented some recommendations of surgical techniques from Chinese experts, and current literatures were reviewed concerning not only UniVATS lobectomy and segmentectomy, but also subxiphoid UniVATS approach and nonintubated UniVATS procedure. This consensus provided a blueprint for the current situation of UniVATS for lung cancer in China.

There were also limitations in this consensus. Only 11 experts have performed lung cancer resection by subxiphoid UniVATS approach, the agreement on this issue was based on the voting result from the 11 experts. Another possible limitation was selection bias, all the experts with the same interests had already completed their learning curves, training surgeons should take this into account.

Looking back in history, the development of minimally invasive thoracic surgery represented the surgeons' constant pursuit of less trauma and faster recovery without compromising on efficacy. Such goals served as the driving force behind the evolution of relevant theories as well as science and technology. Theoretical innovation was reflected in the emerging types of minimally invasive surgery: subxiphoid UniVATS, tube-less UniVATS, even tube-less subxiphoid UniVATS (82), and natural orifice transluminal UniVATS (83). Meanwhile, scientific and technological evolution witnessed the emergence of new medical devices such as hybrid operating room, glasses- 
free $3 \mathrm{D}$ viewing system, fluorescence imaging endoscope, needle-like instruments, holographic projection, and uniportal robotic surgical system (84).

From the survey, we known that over $90 \%$ of the experts in this consensus project agreed that the progress of UniVATS had benefited from the improvement of surgical instruments; $83 \%$ experts considered that UniVATS would be a promising direction of minimally invasive thoracic surgery. As $95 \%$ experts agreed on the demand for randomized controlled trials of UniVATS, prospective studies evaluating long-term outcomes should be conducted to verify the practical value of UniVATS in comparison with multi-portal VATS.

\section{Acknowledgments}

The authors specially thank academician Jie He (Department of Thoracic Surgery, Cancer Hospital, Chinese Academy of Medical Sciences and Peking Union Medical College, Beijing), Prof. Jianxing He (Department of Thoracic Surgery, The First Affiliated Hospital of Guangzhou Medical University, Guangzhou) and Prof. Lunxu Liu (Department of Thoracic Surgery, West China Hospital, Sichuan University, Chengdu) for their great academic support to this project.

The authors also thank the experts for participating in this consensus process (listed by names in alphabetical order): Changli Wang (Department of Lung Cancer, Tianjin Medical University Cancer Institute and Hospital, Tianjin), Daqiang Sun (Department of Thoracic Surgery, Tianjin Chest Hospital, Tianjin), Guangsuo Wang (Department of Thoracic Surgery, Shenzhen People's Hospital, Shenzhen), Haitao Ma (Department of Thoracic Surgery, The First Affiliated Hospital of Soochow University, Suzhou), Haiyu Zhou (Department of Thoracic Surgery, Guangdong Provincial People's Hospital, Guangzhou), Huiyuan Gong (Department of Thoracic Surgery, the First Affiliated Hospital of Bengbu Medical College, Bengbu), Jiaming Che (Department of Thoracic Surgery, Ruijin Hospital, Shanghai Jiao Tong University School of Medicine, Shanghai), Jiang Fan (Department of Thoracic Surgery, Shanghai Pulmonary Hospital, Tongji University School of Medicine, Shanghai), Jie Xiang (Department of Thoracic Surgery, Ruijin Hospital, Shanghai Jiao Tong University School of Medicine, Shanghai), Lei Zhang (Department of Thoracic Surgery, the First Affiliated Hospital of Bengbu Medical College, Bengbu), Lianggang Zhu (Department of Thoracic Surgery, Ruijin Hospital, Shanghai Jiao Tong
University School of Medicine, Shanghai), Lijie Tan (Department of Thoracic Surgery, Zhongshan Hospital, Fudan University, Shanghai), Qiang Tan (Department of Oncological Surgery, Shanghai Chest Hospital, Shanghai Jiao Tong University, Shanghai), Rusong Yang (Department of Thoracic Surgery, Nanjing Chest Hospital, Nanjing), Songtao Xu (Department of Thoracic Surgery, Zhongshan Hospital, Fudan University, Shanghai), Wentao Fang (Department of Thoracic Surgery, Shanghai Chest Hospital, Shanghai Jiao Tong University, Shanghai), Wei Zheng (Department of Thoracic Surgery, Fujian Medical University Union Hospital, Fuzhou), Wenzhao Zhong (Department of Thoracic Surgery, Guangdong Provincial People's Hospital, Guangzhou), Xiaojing Zhao (Department of Thoracic Surgery, Renji Hospital, Shanghai Jiao Tong University School of Medicine, Shanghai), Xueying Yang (Department of Thoracic Surgery, Fourth Affiliated Hospital of China Medical University, Shenyang), Xiangning Fu (Department of Thoracic Surgery, Tongji Hospital, Tongji Medical College, Huazhong University of Science and Technology, Wuhan), Yaxin Shen (Department of Thoracic Surgery, Zhongshan Hospital, Fudan University, Shanghai), Yang Yang (Department of Thoracic Surgery, Shanghai Pulmonary Hospital, Tongji University School of Medicine, Shanghai), Yunhai Yang (Department of Thoracic Surgery, Shanghai Chest Hospital, Shanghai Jiao Tong University, Shanghai), and Zongwu Lin (Department of Thoracic Surgery, Zhongshan Hospital, Fudan University, Shanghai).

Funding: None.

\section{Footnote}

Conflicts of Interest: All authors have completed the ICMJE uniform disclosure form (available at http://dx.doi. org/10.21037/tlcr-20-576). The authors have no conflicts of interest to declare.

Ethical Statement: The authors are accountable for all aspects of the work in ensuring that questions related to the accuracy or integrity of any part of the work are appropriately investigated and resolved.

Open Access Statement: This is an Open Access article distributed in accordance with the Creative Commons Attribution-NonCommercial-NoDerivs 4.0 International License (CC BY-NC-ND 4.0), which permits the noncommercial replication and distribution of the article with 
the strict proviso that no changes or edits are made and the original work is properly cited (including links to both the formal publication through the relevant DOI and the license). See: https://creativecommons.org/licenses/by-nc-nd/4.0/.

\section{References}

1. Rocco G, Martin-Ucar A, Passera E. Uniportal VATS wedge pulmonary resections. Ann Thorac Surg 2004;77:726-8.

2. Fiorelli A, Izzo AC, Arrigo E, et al. Resection of esophageal diverticulum through uniportal video-assisted thoracoscopic surgery. Ann Transl Med 2018;6:179.

3. Gonzalez D, Paradela M, Garcia J, et al. Single-port videoassisted thoracoscopic lobectomy. Interact Cardiovasc Thorac Surg 2011;12:514-5.

4. Duan L, Jiang G, Yang Y. One hundred and fiftysix cases of anatomical pulmonary segmentectomy by uniportal video-assisted thoracic surgery: a 2-year learning experience. Eur J Cardiothorac Surg 2018;54:677-82.

5. Han DP, Xiang J, Jin RS, et al. Uniportal video-assisted thoracic surgery for complicated pulmonary resections. J Vis Surg 2016;2:150.

6. Han DP, Chen K, Zhang YJ, et al. Uniportal video-assisted thoracoscopic combined segmentectomy for lung cancer with incomplete fissure. J Thorac Dis 2017;9:1140-3.

7. Feng M. Dr. Sebastien Gilbert: more evidence is need for the promotion of uniportal VATS in North America. J Thorac Dis 2017;9:E579-80.

8. Bertolaccini L, Batirel H, Brunelli A, et al. Uniportal video-assisted thoracic surgery lobectomy: a consensus report from the Uniportal VATS Interest Group (UVIG) of the European Society of Thoracic Surgeons (ESTS). Eur J Cardiothorac Surg 2019;56:224-9.

9. Sihoe ADL, Han B, Yang TY, et al. The Advent of Ultrahigh Volume Thoracic Surgical Centers in Shanghai. World J Surg 2017;41:2758-68.

10. Liu R, Tan Q, Huang J, et al. Uniportal video assisted thoracic surgery with $2 \mathrm{~cm}$ skin incision for right middle lobectomy with systematic lymphadenectomy. J Thorac Dis 2017;9:4592-6.

11. Liu Z, Yang R, Shao F, et al. Modified procedure of uniportal video-assisted thoracoscopic lobectomy with muscle sparing incision. Ann Transl Med 2016;4:367.

12. Shen Y, Zhang Y, Sun J, et al. Transaxillary uniportal video assisted thoracoscopic surgery for right upper lobectomy. J Thorac Dis 2018;10:E214-7.

13. Zieliński M, Nabialek T, Pankowski J. Transcervical uniportal pulmonary lobectomy. J Vis Surg 2018;4:42 .

14. Stamenovic D, Bostanci K, Messerschmidt A. Posterior uniportal video-assisted thoracoscopic surgery for anatomical lung resections. J Thorac Dis 2017;9:5261-6.

15. Palleschi A, Mendogni P, Mariolo AV, et al. An alternative chest tube placement after uniportal video-assisted thoracic surgery. J Thorac Dis 2018;10:3078-80.

16. Kao CN, Chang CW, Hsieh MC, et al. Pre-embedding subcutaneous suture for chest-tube insertion in uniportal video-assisted thoracoscopic surgery. J Thorac Dis 2017;9:E938-40.

17. Ismail M, Nachira D, Swierzy M, et al. Uniportal videoassisted thoracoscopy major lung resections after neoadjuvant chemotherapy. J Thorac Dis 2018;10:S3655-61.

18. Yang Z, Zhai C. Uniportal video-assisted thoracoscopic surgery following neoadjuvant chemotherapy for locallyadvanced lung cancer. J Cardiothorac Surg 2018;13:33.

19. Li Y, Wang J. Video-assisted thoracoscopic surgery sleeve lobectomy with bronchoplasty: an improved operative technique. Eur J Cardiothorac Surg 2013;44:1108-12.

20. Nwogu CE, Yendamuri S, Demmy TL. Does thoracoscopic pneumonectomy for lung cancer affect survival? Ann Thorac Surg 2010;89:S2102-6.

21. Nagai S, Imanishi N, Matsuoka T, et al. Video-assisted thoracoscopic pneumonectomy: retrospective outcome analysis of 47 consecutive patients. Ann Thorac Surg 2014;97:1908-13.

22. Koryllos A, Stoelben E. Uniportal video-assisted thoracoscopic surgery (VATS) sleeve resections for nonsmall cell lung cancer patients: an observational prospective study and technique analysis. J Vis Surg 2018;4:16.

23. Abu Akar F, Yang C, Lin L, et al. Intra-pericardial double sleeve uniportal video-assisted thoracoscopic surgery left upper lobectomy. J Vis Surg 2017;3:51.

24. Gonzalez-Rivas D, Yang Y, Sekhniaidze D, et al. Uniportal video-assisted thoracoscopic bronchoplastic and carinal sleeve procedures. J Thorac Dis 2016;8:S210-22.

25. Lyscov A, Obukhova T, Ryabova V, et al. Double-sleeve and carinal resections using the uniportal VATS technique: a single centre experience. J Thorac Dis 2016;8:S235-41.

26. Gonzalez-Rivas D, Yang Y, Stupnik T, et al. Uniportal video-assisted thoracoscopic bronchovascular, tracheal and carinal sleeve resectionsdagger. Eur J Cardiothorac Surg 2016;49 Suppl 1:i6-16.

27. Halezeroğlu S. Single incision video-assisted thoracic surgery pneumonectomy for centrally located lung cancer. Future Oncol 2018;14:41-5.

28. Vannucci F, Vieira A, Ugalde PA. The technique of VATS 
right pneumonectomy. J Vis Surg 2018;4:11.

29. Wang BY, Liu CY, Hsu PK, et al. Single-incision versus multiple-incision thoracoscopic lobectomy and segmentectomy: a propensity-matched analysis. Ann Surg 2015;261:793-9.

30. Han KN, Kim HK, Lee HJ, et al. Single-port videoassisted thoracoscopic pulmonary segmentectomy: a report on 30 casesdagger. Eur J Cardiothorac Surg 2016;49 Suppl 1:i42-7.

31. Hernandez-Arenas LA, Purmessur RD, Gonzalez-Rivas D. Uniportal video-assisted thoracoscopic segmentectomy. J Thorac Dis 2018;10:S1205-14.

32. Lin MW, Tseng YH, Lee YF, et al. Computed tomography-guided patent blue vital dye localization of pulmonary nodules in uniportal thoracoscopy. J Thorac Cardiovasc Surg 2016;152:535-544.e2.

33. Park JS, Kim HK, Choi YS, et al. Unplanned conversion to thoracotomy during video-assisted thoracic surgery lobectomy does not compromise the surgical outcome. World J Surg 2011;35:590-5.

34. Sugi K, Sudoh M, Hirazawa K, et al. Intrathoracic bleeding during video-assisted thoracoscopic lobectomy and segmentectomy. Kyobu Geka 2003;56:928-31.

35. Liang C, Wen H, Guo Y, et al. Severe intraoperative complications during VATS Lobectomy compared with thoracotomy lobectomy for early stage non-small cell lung cancer. J Thorac Dis 2013;5:513-7.

36. Gonzalez-Rivas D, Stupnik T, Fernandez R, et al. Intraoperative bleeding control by uniportal video-assisted thoracoscopic surgerydagger. Eur J Cardiothorac Surg 2016;49 Suppl 1:117-24.

37. Abu Akar F, Gonzalez-Rivas D, Fink D. Management of bleeding from an abnormally located S6 arterial branch in a common origin with S2 branch during live surgery. J Vis Surg 2016;2:87.

38. Scarci M, Gonzalez-Rivas D, Schmidt J, et al. Management of Intraoperative Difficulties During Uniportal VideoAssisted Thoracoscopic Surgery. Thorac Surg Clin 2017;27:339-46.

39. Guido Guerrero W, Gonzalez-Rivas D, Hernandez Arenas LA, et al. Techniques and difficulties dealing with hilar and interlobar benign lymphadenopathy in uniportal VATS. J Vis Surg 2016;2:23.

40. Sihoe ADL. Uniportal Lung Cancer Surgery: State of the Evidence. Ann Thorac Surg 2019;107:962-72.

41. Xie D, Wang H, Fei K, et al. Single-port video-assisted thoracic surgery in 1063 cases: a single-institution experiencedagger. Eur J Cardiothorac Surg 2016;49 Suppl 1:131-6.
42. Gao T, Xiang J, Jin R, et al. "Ipsilateral, high, single-hand, sideways"-Ruijin rule for camera assistant in uniportal video-assisted thoracoscopic surgery. J Thorac Dis 2016;8:2952-5.

43. D'Amico TA, Niland J, Mamet R, et al. Efficacy of mediastinal lymph node dissection during lobectomy for lung cancer by thoracoscopy and thoracotomy. Ann Thorac Surg 2011;92:226-31; discussion 231-2.

44. Boffa DJ, Kosinski AS, Paul S, et al. Lymph node evaluation by open or video-assisted approaches in 11,500 anatomic lung cancer resections. Ann Thorac Surg 2012;94:347-53; discussion 353.

45. Lin Z, Xi J, Xu S, et al. Uniportal video-assisted thoracic surgery lobectomy in semiprone position: primary experience of 105 cases. J Thorac Dis 2015;7:2389-95.

46. Liu C, Ma L, Guo C, et al. Non-grasping en bloc mediastinal lymph node dissection through uniportal video-assisted thoracic surgery for lung cancer surgery. J Thorac Dis 2016;8:2956-9.

47. Cai Y, Han Y, Zhang N, et al. Modular Uniportal Video-Assisted Thoracoscopic Lobectomy and Lymphadenectomy: A Novel Pattern of Endoscopic Lung Cancer Resection. J Laparoendosc Adv Surg Tech A 2017;27:1230-5.

48. Hernandez-Arenas LA, Lin L, Purmessur RD, et al. Uniportal video-assisted thoracoscopic early learning curve for major lung resections in a high volume training center. J Thorac Dis 2018;10:S3670-7.

49. Nachira D, Meacci E, Porziella V, et al. Learning curve of uniportal video-assisted lobectomy: analysis of 15 -month experience in a single center. J Thorac Dis 2018;10:S3662-9.

50. Liu X, Chen X, Shen Y, et al. Learning curve for uniportal video-assisted thoracoscopic surgery lobectomy-results from 120 consecutive patients. J Thorac Dis 2018;10:5100-7.

51. Bedetti B, Bertolaccini L, Solli P, et al. Learning curve and established phase for uniportal VATS lobectomies: the Papworth experience. J Thorac Dis 2017;9:138-42.

52. Cheng K, Zheng B, Zhang S, et al. Feasibility and learning curve of uniportal video-assisted thoracoscopic segmentectomy. J Thorac Dis 2016;8:S229-34.

53. Gezer S, Avci A, Turktan M. Cusum analysis for learning curve of videothoracoscopic lobectomy. Open Med (Wars) 2016;11:574-7.

54. Abouarab AA, Rahouma M, Kamel M, et al. Single Versus Multi-Incisional Video-Assisted Thoracic Surgery: A Systematic Review and Meta-analysis. J Laparoendosc Adv Surg Tech A 2018;28:174-85. 
55. Hirai K, Takeuchi S, Usuda J. Single-incision thoracoscopic surgery and conventional video-assisted thoracoscopic surgery: a retrospective comparative study of perioperative clinical outcomesdagger. Eur J Cardiothorac Surg 2016;49 Suppl 1:i37-41.

56. Xu GW, Xiong R, Wu HR, et al. A prospective comparative study examing the impact of uniportal and three portal video-assisted thoracic surgery on short-term quality of life in lung cancer. Zhonghua Wai Ke Za Zhi 2018;56:452-7.

57. Gonzalez-Rivas D, Paradela M, Fernandez R, et al. Uniportal video-assisted thoracoscopic lobectomy: two years of experience. Ann Thorac Surg 2013;95:426-32.

58. Hsu PK, Lin WC, Chang YC, et al. Multiinstitutional analysis of single-port video-assisted thoracoscopic anatomical resection for primary lung cancer. Ann Thorac Surg 2015;99:1739-44.

59. Liu CC, Shih CS, Pennarun N, et al. Transition from a multiport technique to a single-port technique for lung cancer surgery: is lymph node dissection inferior using the single-port technique? dagger. Eur J Cardiothorac Surg 2016;49 Suppl 1:i64-72.

60. Ng CS, Kim HK, Wong RH, et al. Single-Port VideoAssisted Thoracoscopic Major Lung Resections: Experience with 150 Consecutive Cases. Thorac Cardiovasc Surg 2016;64:348-53.

61. Han KN, Kim HK, Choi YH. Midterm outcomes of single port thoracoscopic surgery for major pulmonary resection. PLoS One 2017;12:e0186857.

62. Shen Y, Wang H, Feng M, et al. Single- versus multipleport thoracoscopic lobectomy for lung cancer: a propensity-matched studydagger. Eur J Cardiothorac Surg 2016;49 Suppl 1:i48-53.

63. Chung JH, Choi YS, Cho JH, et al. Uniportal videoassisted thoracoscopic lobectomy: an alternative to conventional thoracoscopic lobectomy in lung cancer surgery? Interact Cardiovasc Thorac Surg 2015;20:813-9.

64. Zhu Y, Liang $\mathrm{M}, \mathrm{Wu} W$, et al. Preliminary results of single-port versus triple-port complete thoracoscopic lobectomy for non-small cell lung cancer. Ann Transl Med 2015;3:92.

65. Mu JW, Gao SG, Xue Q, et al. A Matched Comparison Study of Uniportal Versus Triportal Thoracoscopic Lobectomy and Sublobectomy for Early-stage Nonsmall Cell Lung Cancer. Chin Med J (Engl) 2015;128:2731-5.

66. Chang JM, Kam KH, Yen YT, et al. From biportal to uniportal video-assisted thoracoscopic anatomical lung resection: A single-institute experience. Medicine
(Baltimore) 2016;95:e5097.

67. Hirai K, Usuda J. Current status and issues of uniportal video-assisted thoracic surgery in Japan. J Vis Surg 2020;6:27.

68. Suda T, Sugimura H, Tochii D, et al. Single-port thymectomy through an infrasternal approach. Ann Thorac Surg 2012;93:334-6.

69. Liu CC, Wang BY, Shih CS, et al. Subxiphoid singleincision thoracoscopic left upper lobectomy. J Thorac Cardiovasc Surg 2014;148:3250-1.

70. Aresu G, Weaver H, Wu L, et al. The Shanghai Pulmonary Hospital uniportal subxiphoid approach for lung segmentectomies. J Vis Surg 2016;2:172.

71. Aresu G, Weaver H, Wu L, et al. Uniportal subxiphoid video-assisted thoracoscopic bilateral segmentectomy for synchronous bilateral lung adenocarcinomas. J Vis Surg 2016;2:170.

72. Gonzalez-Rivas D, Lirio F, Sesma J, et al. Subxiphoid complex uniportal video-assisted major pulmonary resections. J Vis Surg 2017;3:93.

73. Aresu G, Wu L, Lin L, et al. The Shanghai Pulmonary Hospital subxiphoid approach for lobectomies. J Vis Surg 2016;2:135.

74. Song N, Zhao DP, Jiang L, et al. Subxiphoid uniportal video-assisted thoracoscopic surgery (VATS) for lobectomy: a report of 105 cases. J Thorac Dis 2016;8:S251-7.

75. Pompeo E, Mineo D, Rogliani P, et al. Feasibility and results of awake thoracoscopic resection of solitary pulmonary nodules. Ann Thorac Surg 2004;78:1761-8.

76. Rocco G, Romano V, Accardo R, et al. Awake singleaccess (uniportal) video-assisted thoracoscopic surgery for peripheral pulmonary nodules in a complete ambulatory setting. Ann Thorac Surg 2010;89:1625-7.

77. Ahn S, Moon Y, AlGhamdi ZM, et al. Nonintubated Uniportal Video-Assisted Thoracoscopic Surgery: A Single-Center Experience. Korean J Thorac Cardiovasc Surg 2018;51:344-9.

78. Wang ML, Galvez C, Chen JS, et al. Non-intubated singleincision video-assisted thoracic surgery: a two-center cohort of 188 patients. J Thorac Dis 2017;9:2587-98.

79. Peng G, Liu M, Luo Q, et al. Spontaneous ventilation anesthesia combined with uniportal and tubeless thoracoscopic lung biopsy in selected patients with interstitial lung diseases. J Thorac Dis 2017;9:4494-501.

80. Rocco G. Non-intubated uniportal lung surgerydagger. Eur J Cardiothorac Surg 2016;49 Suppl 1:13-5.

81. Bedetti B, Patrini D, Bertolaccini L, et al. Uniportal non- 
intubated thoracic surgery. J Vis Surg 2018;4:18.

82. Liu Z, Yang R, Sun Y. Non-intubated subxiphoid uniportal video-assisted thoracoscopic thymectomy. Interact Cardiovasc Thorac Surg 2019;29:742-5.

83. $\mathrm{Li} \mathrm{Z,} \mathrm{Ng} \mathrm{CS.} \mathrm{Future} \mathrm{of} \mathrm{uniportal} \mathrm{video-assisted}$

Cite this article as: Han D, Cao Y, Wu H, Wang H, Jiang L, Zhao D, Yao F, Li S, Zhang C, Zheng B, Fan J, Liao Y, Qiu B, Tan F, Chen C, Zhu Y, Gao S, Li H. Uniportal video-assisted thoracic surgery for the treatment of lung cancer: a consensus report from Chinese Society for Thoracic and Cardiovascular Surgery (CSTCVS) and Chinese Association of Thoracic Surgeons (CATS). Transl Lung Cancer Res 2020;9(4):971-987. doi: 10.21037/tlcr-20-576 thoracoscopic surgery-emerging technology. Ann Cardiothorac Surg 2016;5:127-32.

84. Rocco R, Rocco G. Future study direction on single port (uniportal) VATS. J Thorac Dis 2016;8:S328-32. 